\title{
The influence of a simple blood transfusion policy on overtransfusion in acute upper gastrointestinal haemorrhage 䇺
}

\author{
Authors: Adam Stokes, ${ }^{A}$ Clare Thompson, ${ }^{B}$ Andrew Clegg $^{C}$ and Jonathon Snook ${ }^{D}$
}

\begin{abstract}
Blood transfusion is widely used in the management of acute upper gastrointestinal haemorrhage (AUGIH). Trial data suggests that excessive transfusion may be detrimental, yet overtransfusion remains commonplace. This study reports the impact of introducing a simple cross-match policy in a district general hospital, which resulted in a substantial fall in the prevalence of overtransfusion (odds ratio $0.43 ; 95 \%$ confidence interval $0.19-0.98$ ), with potential patient benefits in terms of rebleeding, and a reduction in the total blood transfused from 162 to 121 units per 100 patients with AUGIH. For the cost of blood alone, this corresponds to projected savings across the NHS in England in excess of $€ 2$ million per annum.
\end{abstract}

KEYWORDS: Acute upper gastrointestinal haemorrhage, blood transfusion, overtransfusion

\section{Introduction}

Acute upper gastrointestinal haemorrhage (AUGIH) is a common and potentially serious medical emergency, with an incidence of about 1 per 1,000 population per annum, and a 28 -day mortality of $8-10 \% \cdot{ }^{1-3}$ While bleeding ceases spontaneously in the majority, rebleeding occurs in $10-15 \%$ of cases despite pharmacological and/or endoscopic intervention. ${ }^{2,4}$ Rebleeding is a major predictor of poor prognosis in AUGIH. ${ }^{5}$

A recent UK national audit revealed that $44 \%$ of individuals with AUGIH receive a blood transfusion within 12 hours of admission, ${ }^{6}$ and it is estimated that $14 \%$ of all blood used in the UK is for the treatment of gastrointestinal bleeding. ${ }^{7}$ Transfusion seems an intuitive aspect of management, and in the exsanguinating patient it may be lifesaving, however this is a relatively uncommon situation. On the other hand, there are already many reasons for caution in the use of blood transfusion, including the risk of immunological, infective and metabolic complications. ${ }^{8}$ Furthermore, blood is a valuable

Authors: AST3 in medicine, Poole Hospital NHS Foundation Trust, Poole, UK; ${ }^{B}$ associate transfusion practitioner, Poole Hospital NHS Foundation Trust, Poole, UK; ' visiting professor, University of Southampton, Southampton, UK; ${ }^{D}$ consultant physician, Poole Hospital NHS Foundation Trust, Poole, UK

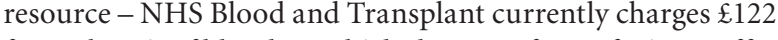
for each unit of blood, to which the cost of transfusion staff time, equipment and consumables must be added.

Over and above these concerns, retrospective studies have suggested that early transfusion is associated with a substantially increased risk of re-bleeding and consequent mortality in patients with AUGIH. ${ }^{2,4,9}$ Caution is required in the interpretation of these findings because bleed severity is a major potential confounding factor - patients with severe bleeds are inherently more likely to receive both a blood transfusion and have an adverse outcome. However, two randomised controlled trials now support the suspicion of a causal relationship between transfusion and outcome, by providing strong evidence that a restrictive blood transfusion policy improves the prognosis in severe AUGIH. ${ }^{10,11}$

This finding leads to the concept of 'overtransfusion', a situation where blood is administered in excess of requirements, with the immediate potential for deleterious effects. ${ }^{12}$

There is no agreed definition, but taking a post-transfusion haemoglobin concentration ([Hb]) of greater than $100 \mathrm{~g} / \mathrm{L}$ as an arbitrary marker, in-house audits in 2008 and 2011 revealed that overtransfusion was commonplace - about $50 \%$ of subjects with AUGIH given blood had a post-transfusion [Hb] of over $100 \mathrm{~g} / \mathrm{L}$, and in $16 \%$ it exceeded $120 \mathrm{~g} / \mathrm{L}$.

The post-transfusion $[\mathrm{Hb}]$ only allows overtransfusion to be identified in retrospect, when it is too late to intervene. However, the audits suggested that a high pre-transfusion $[\mathrm{Hb}]$ and a cross-match request for a large number of units were both major predictors of subsequent overtransfusion. As a first step towards attempting to address the issue of overtransfusion, the Gastroenterology Department at Poole Hospital introduced guidelines for blood use in patients with AUGIH in the form of a simple table as outlined below. This study describes the impact of these guidelines.

\section{Method}

A simple cross-match policy was devised aiming to limit the number of units initially provided for patients with AUGIH according to the pre-transfusion $[\mathrm{Hb}]$ and presence or absence of shock and/or suspected varices (Table 1). The target in patients transfused was a post-transfusion $[\mathrm{Hb}]$ in the range 90-100 g/L. Overtransfusion was arbitrarily defined as a post-transfusion [Hb] exceeding $100 \mathrm{~g} / \mathrm{L}$, while figures of over $110 \mathrm{~g} / \mathrm{L}$ were considered to indicate major overtransfusion. 
Table 1. Poole cross-match policy guidelines.

\begin{tabular}{lll}
$\begin{array}{l}\text { Pre-transfusion } \\
\text { [Hb], g/L }\end{array}$ & \multicolumn{2}{c}{ Units for cross-match } \\
\cline { 2 - 3 } & $\begin{array}{l}\text { Not shocked and } \\
\text { varices not suspected }\end{array}$ & $\begin{array}{l}\text { Shocked and/or } \\
\text { varices suspected }\end{array}$ \\
$\geq 100$ & 0 & 0 \\
$90-9$ & 0 & 2 \\
$80-9$ & $(1)^{\mathrm{a}}$ & 3 \\
$70-9$ & 2 & 4 \\
$60-9$ & 3 & 5 \\
$<60$ & 4 & 6 \\
\hline
\end{tabular}

aIf a one unit transfusion felt to be justified. [Hb] = haemoglobin concentration.

After discussion, refinement and a period of staff education, the policy was introduced in Poole Hospital in June 2012. The guidelines were 'policed' by the biomedical scientist on shift, with the option of involvement of the on-call consultant endoscopist if an agreement could not be reached with the requesting clinician. Clinicians were free to request more blood at a later date if required, in particular due to a falling [ $\mathrm{Hb}]$ resulting from ongoing bleeding.

Anonymised demographic and clinical data were collected retrospectively for all patients referred for endoscopy at Poole Hospital with suspected AUGIH during two six-month periods, the first before (July to December 2011 - group 1) and the second after (July to December 2012 - group 2) introduction of the policy. Exclusion criteria were (1) lack of confirmation of an acute bleed, (2) inadequate clinical information and (3) extreme case complexity.

The number of units cross-matched and transfused was assessed from hospital records and confirmed using the transfusion laboratory computer system, which records the fate of each unit of blood. This enabled identification of the exact date and time that the blood was transfused so that $[\mathrm{Hb}]$ results before and after transfusion could be identified. This was especially useful in those situations where a patient had a cross- match requested, but was not transfused until a later date. In situations where a patient required more than one transfusion, only the first episode was recorded.

Statistical analyses were undertaken to assess (1) the effect of the cross-match guidelines on blood usage and the proportion of patients overtransfused, (2) compliance with the cross-match policy guidelines for AUGIH and (3) clinical factors predictive of overtransfusion in both cohorts. Specifically, comparisons were made between the two patient groups in terms of their baseline characteristics and the different outcome measures. Continuous data were summarised using the mean with $95 \%$ confidence intervals (CIs) or median with interquartile range, and compared using either a t-test or rank-sum test (depending on the probability distribution of the data). Categorical data were presented as proportions and the groups compared using the Pearson $\chi^{2}$ and, where appropriate, odds ratios with 95\% CIs. Multiple logistic regression analysis was used to assess the patient characteristics and clinical factors that might predict the odds of overtransfusion. Regression models were developed iteratively using least squares approach and assessed through analysis of variance and residual plots to provide an appropriately specified and parsimonious model. All analyses were undertaken using STATA software (version 13.1). Data were not available for every variable for all patients, and these observations were excluded from the subsequent analyses that included the particular variable.

\section{Results}

A total of 245 subjects were initially identified. Exclusions were made as follows: AUGIH not confirmed on review (11 cases); clinical information inadequate (7 cases); and clinical complexity (1 case). This left 122 subjects for analysis in group 1 and 105 in group 2. There were no significant differences between the two groups in age, sex ratio, $[\mathrm{Hb}]$ at presentation or Rockall score (Table 2).

A total of 259 units and 148 units were cross-matched for the patients in groups 1 and 2 respectively, representing a $43 \%$ reduction. Following on from this, a total of 198 units and 127 units were transfused, a $36 \%$ reduction. The reduction in

Table 2. Comparison of the baseline characteristics of the two patient cohorts.

\begin{tabular}{llll} 
Baseline characteristics & Group 1 (2011) & Group 2 (2012) & p value \\
Total in group & 131 & 114 & - \\
Included in analysis & 122 & 105 & - \\
Median age (IQR), years & $78(66-85)$ & $75(66-84)$ & 0.45 \\
Male & $57 \%$ & $50 \%$ & - \\
Confirmed varices & $7 \%$ & $9 \%$ & 0.89 \\
Median presentation [Hb] (IQR), g/L & $96(73-121)$ & $92(72-124)$ & 0.18 \\
Rockall score & & & $8 \%$ \\
$\quad 0-1$ & $5 \%$ & $27 \%$ & $47 \%$ \\
$\quad 2-3$ & $26 \%$ & $14 \%$ & $4 \%$ \\
\hline $4-5$ & $41 \%$ & & \\
$8+7$ & $25 \%$ & $3 \%$ & \\
\hline
\end{tabular}

$[\mathrm{Hb}]=$ haemoglobin concentration; $\mathrm{IQR}=$ interquartile range. 
Table 3. The usage of blood in each cohort.

$\begin{array}{lll}\text { Characteristics } & \begin{array}{l}\text { Group 1 } \\ (\mathbf{2 0 1 1 )}\end{array} & \begin{array}{l}\text { Group 2 } \\ \text { (2012) }\end{array} \\ \text { Cases analysed } & 122 & 105 \\ \text { Units cross-matched in total } & 259 & 148 \\ \text { Units transfused in total } & 198 & 127 \\ \text { Cross-matched, \% (n) } & 64(78) & 51(54) \\ \text { Units cross-matched per patient, n } & 3.3(259 / 78) & 2.7(148 / 54) \\ \text { Transfused, \% (n) } & 58(71) & 50(53) \\ \text { Transfused, } \mathrm{n} & 2.8(198 / 71) & 2.4(127 / 53)\end{array}$

blood transfused was due to the combination of (1) a slight disparity between the number of subjects in each group (122 vs $105),(2)$ a reduction in the proportion of patients transfused $\left(58 \%\right.$ vs $\left.50 \% ; \chi^{2}=1.36, p=0.24\right)$, and ( 3 ) a reduction in the number of units administered to each recipient of blood (mean 2.8 vs 2.4 ; t-test $1.95, \mathrm{p}=0.05$ ). The data are shown in Table 3.

Using the definitions given in the method section, the proportion of patients overtransfused (as a percentage of those transfused) decreased from $48 \%$ in group 1 to $28 \%$ in group 2 . This was primarily due to a significant reduction in the proportion receiving a major overtransfusion $(>110 \mathrm{~g} / \mathrm{L})$, which fell from $22 \%$ to $8 \%$ (Table 4 ). Logistic regression analysis of combined data from the two cohorts confirmed that 'initial $[\mathrm{Hb}]$ ' and 'units transfused' were the two major independent clinical variables predictive of overtransfusion - with odds ratios (95\% CI) of 1.10 (1.04-1.16) and 2.87 (1.61-5.11) respectively.

Compliance with the guidelines was assessed for group 2, and compared with the theoretical compliance for group 1, had the guidelines existed in 2011. There was a significant reduction in the proportion of non-compliant cases from $37 \%$ in group 1 to $22 \%$ in group 2, primarily due to a fall in the prevalence of more profound non-compliance (Table 5).

Data for length of stay, rebleeding and 28-day mortality are shown in Table 6. As anticipated, blood transfusion was associated with longer lengths of stay, and increased risks of rebleeding and death. However, it is important to stress that the study was not powered to detect differences in these outcome parameters between the two groups, and that the differences were not statistically significant. Furthermore, the retrospective study design is likely to result in an underestimate of rebleeding rates.

At Poole Hospital, the Departments of Medicine and Elderly Care manage patients with AUGIH. Independent data from the Transfusion Service were used to assess the impact of the cross-match policy by comparing the total quantity of blood

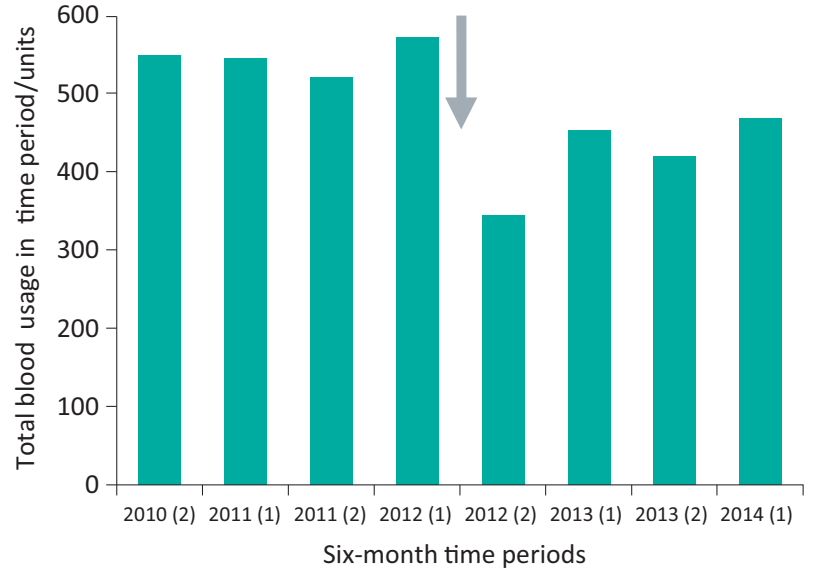

Fig 1. Total blood usage in the Departments of Medicine and Elderly Care, Poole Hospital 2010-2014. Grey arrow shows the point of introduction of the cross-match policy in 2012.

transfused for all indications by these two departments for the 2 years before and after introduction (Fig 1). The figure fell from a mean of 91.1 units transfused per month for July 2010 to June 2012, to a mean of 70.2 units per month for July 2012 to June 2014 (t-test 4.25, p=0.01).

\section{Conclusion}

The link between rebleeding and the increased risk of morbidity and mortality in AUGIH is well established. This has rightly resulted in increasing emphasis on active measures to achieve stable haemostasis in AUGIH over recent years by means of pharmacological, endoscopic and radiological interventions. ${ }^{3,13,14}$ Nevertheless, blood transfusion has been an integral component of the management of AUGIH for decades, on the grounds that it would seem logical to replace the body fluid lost on a like for like basis.

However, two randomised controlled trials have concluded that excessive transfusion may actually be detrimental in severe AUGIH, in particular by predisposing to rebleeding. In the first of these, ${ }^{10}$ a small study, the rebleed rate was $9 / 24$ (37.5\%) in the group receiving at least 2 units of blood in the first 24 hours, but just $1 / 26(3.8 \%)$ in the group restricted to transfusion only if profoundly anaemic or shocked $(\mathrm{p}<0.01)$. A difference in rebleed rates was confirmed in the second study. ${ }^{11}$ This had sufficient power to allow a comparison of 45-day mortality rates between groups allocated to a liberal strategy (transfusion when $[\mathrm{Hb}$ ] below $90 \mathrm{~g} / \mathrm{L}$ ) or a restrictive strategy (transfusion when $[\mathrm{Hb}$ ] below $70 \mathrm{~g} / \mathrm{L})$ - with figures of $41 / 445(9.2 \%)$ and 23/444 (5.2\%) respectively $(\mathrm{p}<0.02)$.

Table 4. Overtransfusion in each group (see text for definitions).

\begin{tabular}{llll} 
Post-transfusion $[\mathrm{Hb}]$ & Group 1 (2011), \% (n) & Group 2 (2012), \% (n) & OR (95\% CI) \\
$>100 \mathrm{~g} / \mathrm{L}$ & $48\left(33 / 69^{a}\right)$ & $28(15 / 53)$ & $0.43(0.19-0.98)$ \\
$>110 \mathrm{~g} / \mathrm{L}$ & $22\left(15 / 69^{a}\right)$ & $8(4 / 53)$ & $0.29(0.07-1.02)$ \\
\hline
\end{tabular}

aTwo subjects in group 1 had no recorded post-transfusion [Hb], and could not be assessed for overtransfusion. $\mathrm{CI}=$ confidence interval; $[\mathrm{Hb}]=$ haemoglobin concentration; $\mathrm{OR}=$ odds ratio. 
Table 5. Non-compliance with the cross-match policy.

\begin{tabular}{llll}
\hline Outside guidelines & Group 1 (2011), \% (n) & Group 2 (2012), \% (n) & OR (95\% CI) \\
By $\geq 1$ units & $37(45 / 122)$ & $22(23 / 105)$ & $0.47(0.24-0.89)$ \\
By $\geq 2$ units & $27(33 / 122)$ & $10(11 / 105)$ & $0.32(0.14-0.69)$ \\
\hline CI $=$ confidence interval; OR = odds ratio. & & &
\end{tabular}

These observations are at first sight counterintuitive, but studies of acute haemorrhage in human trauma ${ }^{15}$ and experimental animals ${ }^{16}$ have revealed comparable findings, and provided some insight into the pathophysiological basis for it. In essence, the adaptive response to acute haemorrhage from any source involves a number of mechanisms directed towards minimising blood loss, including the development of hypotension, vasoconstriction and a hypercoagulable state. In evolutionary terms this presumably conveys survival advantage. Blood transfusion opposes all of these changes, ${ }^{15,16}$ and it is likely that the same applies to bleeding in the specific context of AUGIH. ${ }^{10}$

The trial findings suggest that the relationship between posttransfusion $[\mathrm{Hb}]$ and the probability of an adverse outcome in severe AUGIH may be a U-shaped curve with undertransfusion potentially leading to an increased risk of cardiovascular complications, and excessive transfusion resulting in an increased likelihood of rebleeding. This introduces the concept of 'overtransfusion'. While the optimal post-transfusion [Hb] may of course vary depending on other clinical factors, trial data ${ }^{10,11}$ and national guidelines ${ }^{3,13}$ would suggest that is likely to be in the range $70-90 \mathrm{~g} / \mathrm{L}$.

Overtransfusion is not only potentially detrimental for the reasons outlined above, but also clearly wasteful of a valuable resource. Our study suggests that the introduction of a simple cross-match policy can curb the risk of overtransfusion, with a reduction in the transfusion rate from 162 to 121 units per 100 patients with AUGIH. A typical District General Hospital managing 250 cases a year could therefore potentially save $\mathfrak{1 2 , 0 0 0}$ pa on blood alone - if applied across the NHS in England this equates to over $\mathfrak{£} 2$ million pa. In addition, one might anticipate considerable indirect cost savings from a reduction in the interventions and extended lengths of stay required to address rebleeding episodes.

There may be a number of reasons why overtransfusion is so common. First, transfusion to achieve a haemoglobin level of over $100 \mathrm{~g} / \mathrm{L}$ has been standard medical practice for decades, so it is likely to take a while to alter this mindset. Second, quantifying blood loss at the bedside can be difficult, and there is an understandable tendency to err on the side of 'caution', with allowance made for fluid re-equilibriation. Third, decisions regarding cross-matching and transfusion are often made by relatively inexperienced junior medical staff, sometimes in emotionally charged situations. Finally, we are not aware of any other specific cross-matching guidelines for AUGIH, either published or in use in UK hospitals, and contemporary national and international guidelines on this topic are worded in somewhat vague terms. ${ }^{3,13,14}$ The 2007 countrywide audit ${ }^{6}$ confirmed that the use of blood for AUGIH at Poole Hospital adjusted for bleed severity was close to the national average, indicating that our findings are likely to be a fair reflection of the national pattern of blood usage.

Our results may have underestimated the potential savings from a cross-match policy for two particular reasons. First, compliance with the guidelines in group 2 was not perfect. Individual case investigations are underway to establish the reasons for this, and it may be that in some cases there were extenuating circumstances. Equally, with ongoing education it may be that compliance - and therefore savings - can be improved further. Second, to avoid any possibility that our patients might end up being undertransfused, we chose thresholds for the cross-match policy and the definition of overtransfusion on the basis of trial results ${ }^{10,11}$ and national guidelines ${ }^{3,13}$ could be deemed generously high. With further experience and consensus, it is likely that both could be safely lowered.

Many other factors may of course have contributed to the fall in departmental blood usage for all indications shown in

Fig 1. Nevertheless the step-wise reduction in usage at the time of introduction of our cross-match policy is quite striking. Interestingly, the mean reduction of just over 20 units per month is rather larger than the fall of approximately 12 units per month that we would have predicted from the results of the study. This may simply reflect the influence of unrelated initiatives, ${ }^{17}$ but an alternative interpretation could be that the introduction of our specific cross-match policy for AUGIH encouraged medical staff to adopt a more conservative approach to transfusion in other clinical circumstances.

It is important to highlight that the study reported here is observational, and not a randomised controlled trial. We did

Table 6. Outcome parameters.

\begin{tabular}{llllll} 
Group & Transfused? & $\mathbf{n}$ & Median LoS (IQR), days & Rebleed (\%) & 28-day mortality (\%) \\
Group 1 (2011) & No & 51 & $3(2-5)$ & $0(0.0)$ & $2(3.9)$ \\
& Yes & 71 & $8(4-13)$ & $3(5.6)$ & $10(14.1)$ \\
Group 2 (2012) & No & 52 & $2(1-5)$ & $1(1.9)$ & $1(1.9)$ \\
& Yes & 53 & $10(5-15)$ & $3(5.7)$ & $5(9.4)$ \\
\hline IQR = interquartile range; LoS = length of stay.
\end{tabular}


our best to minimise other influences, for example by using the same calendar months for each cohort to control for the effect of junior doctor experience, and concluding the second study period just before the month of publication of the seminal NEJM paper. ${ }^{11}$ We cannot however exclude the possibility that external factors may have influenced the results, notably the drive towards more appropriate use of blood transfusion. ${ }^{17}$ It was an unfortunate coincidence that the NICE guidelines for management of acute upper GI bleed ${ }^{14}$ were published in the same month as we introduced our cross-match policy. However, we feel that these weighty guidelines are unlikely to have influenced our results, as the advice regarding blood transfusion simply encourages clinicians to 'base decisions on blood transfusion on the full clinical picture, recognising that overtransfusion may be as damaging as undertransfusion'. ${ }^{14}$

In conclusion, we have demonstrated that introducing a simple cross-match policy for AUGIH can considerably reduce the usage of blood for this indication and the prevalence of overtransfusion. On the basis of published trial data, further patient benefits and savings would be anticipated as the result of a reduced risk of rebleeding. We would encourage other units to trial the cross-match policy and publish their prospective analysis of the outcome.

\section{Acknowledgements}

The study was conceived by AS, CT and JAS, and data collected by AS and CT. AS and JAS drafted the paper, and all authors approved the final version. We are indebted to Dr Nicholas Sharer for his critical appraisal of the manuscript.

\section{References}

1 Blatchford O, Davidson LA, Murray WR, Blatchford M, Pell J. Acute upper gastrointestinal haemorrhage in west of Scotland: case ascertainment study. BMJ 1997;315:510-4.

2 Hearnshaw S, Logan RFA, Palmer KR et al. Outcomes following early red blood cell transfusion in acute upper gastrointestinal bleeding. Aliment Pharmacol Ther 2010;32:215-24.

3 Barkun N, Bardou M, Kuipers EJ et al. International consensus recommendations on the management of patients with non-variceal upper gastrointestinal bleeding. Ann Intern Med 2010;152:101-13.
4 Restellini S, Kherad O, Jairath V, Martel M, Barkun AN. Red blood cell transfusion is associated with increased rebleeding in patients with non-variceal upper gastrointestinal bleeding. Aliment Pharmacol Ther 2013;37:316-22.

5 Zimmerman J, Siguencia J, Tsvang E, Beeri R, Arnon R. Predictors of mortality in patients admitted to hospital for acute upper gastrointestinal hemorrhage. Scand J Gastroenterol 1995;30:327-31.

6 British Society of Gastroenterology. UK comparative audit of upper gastrointestinal bleeding and the use of blood. UK Upper GI Bleeding Audit, 2007. Available online at www.bsg.org.uk/clinical/general/ uk-upper-gi-bleeding-audit.html [Accessed 9 December 2014]

7 Wallis JP, Wells AW, Chapman CE. Changing indications for red cell transfusion from 2000 to 2004 in the North of England. Transfus Med 2006;16:411-7.

8 Klein HG, Spahn DR, Carson JL. Red blood cell transfusion in clinical practice. Lancet 2007;370: 415-26.

9 Taha AS, McCloskey C, Craigen T et al. Mortality following blood transfusion for non-variceal upper gastrointestinal bleeding. Frontline Gastroenterol 2011;2:218-25.

10 Blair S, Janvrin SB, McCollum CN, Greenhalgh RM. Effect of early blood transfusion on gastrointestinal haemorrhage. Br J Surg 1986;73:783-5.

11 Villanueva C, Colomo A, Bosch A et al. Transfusion strategies for acute upper gastrointestinal bleeding. N Engl J Med 2013;368; 1:11-21.

12 Duggan JM. Gastrointestinal haemorrhage: should we transfuse less? Dig Dis Sci 2009;54:1662-6.

13 SIGN. Management of acute upper and lower gastro-intestinal bleeding - a national clinical guideline. Edinburgh: Scottish Intercollegiate Guidelines Network, 2008.

14 UK National Clinical Guideline Centre. Acute upper gastrointestinal bleeding: management. NICE clinical guideline 141. London: National Institute for Health and Care Excellence, 2012.

15 Bickell WH, Wall MJ, Pepe PE et al. Immediate versus delayed fluid resuscitation for hypotensive patients with penetrating torso injuries. N Engl J Med 1994;331:1105-9.

16 Shaftan GW, Chiu CJ, Dennis C, Harris B. Fundamentals of physiologic control of arterial hemorrhage. Surgery 1965;58:851-6.

17 Murphy MF, Waters JH, Wood EM, Yazer MH. Transfusing blood safely and appropriately. BMJ 2013;347:29-33.

Address for correspondence: Dr J Snook, Poole Hospital NHS Foundation Trust, Longfleet Road, Poole BH15 2JB, UK. Email: jonathon.snook@poole.nhs.uk

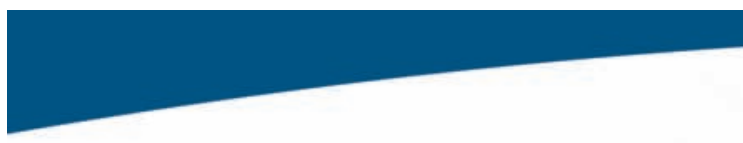

Join the discussion online

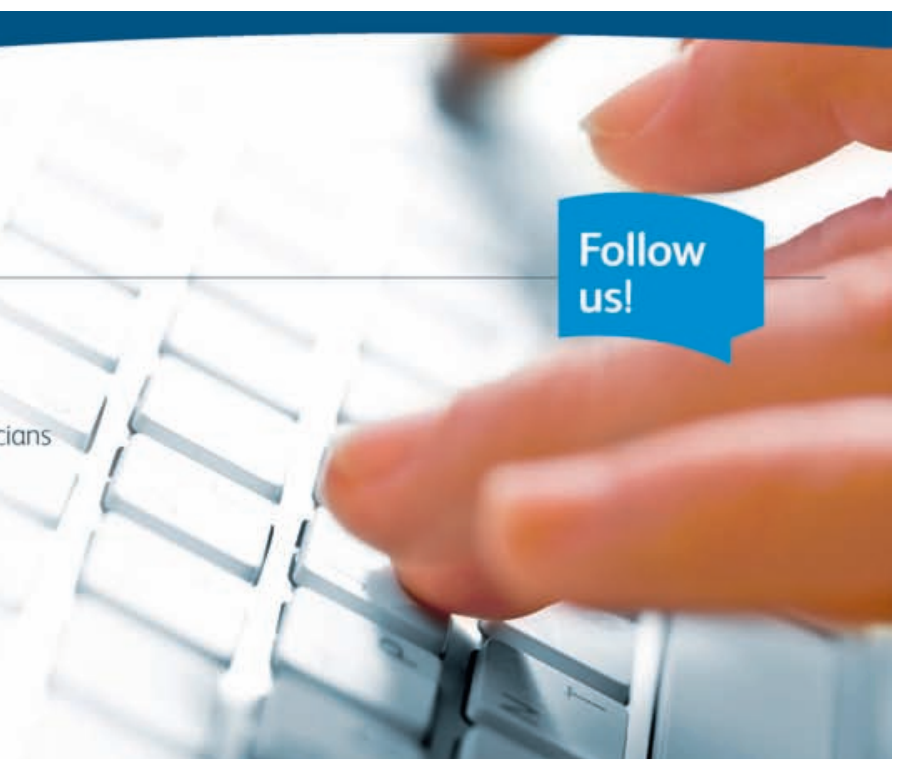

